\title{
Identidad, frontera y oportunidades. Los Ye'kwana de Venezuela en la frontera con Brasil
}

Identity, border, and opportunities. The Ye'kuana of Venezuela at the border with Brazil

\section{Nalúa Rosa Silva Monterrey}

\section{(2) OpenEdition}

\section{Journals}

Edición electrónica

URL: http://journals.openedition.org/aa/3156

DOI: 10.4000/aa.3156

ISSN: 2357-738X

Editor

Programa de Pós-Graduação em Antropologia Social (UnB)

Edición impresa

Fecha de publicación: 1 diciembre 2018

Paginación: 27-54

ISSN: 0102-4302

Referencia electrónica

Nalúa Rosa Silva Monterrey, «Identidad, frontera y oportunidades. Los Ye'kwana de Venezuela en la frontera con Brasil», Anuário Antropológico [En línea], v.43 n.2 | 2018, Publicado el 26 mayo 2019, consultado el 27 abril 2021. URL: http://journals.openedition.org/aa/3156 ; DOI: https://doi.org/ 10.4000/aa.3156

\section{(c) $(1)(9)$}

Anuário Antropológico is licensed under a Creative Commons Atribuição-Uso Não-Comercial-Proibição de realização de Obras Derivadas 4.0 International. 


\title{
Identidad, frontera y oportunidades. Los Ye'kwana de Venezuela en la frontera con Brasil
}

\author{
Nalúa Rosa Silva Monterrey
}

CIAG-UNEG

\section{Introducción}

A partir de nuestra experiencia como investigadora entre los Ye'kwana de la Cuenca del Caura, desde hace más de veinticinco años, y de las fuentes documentales, analizaremos brevemente las formas de organización de los Ye'kwana y las relaciones intra e interétnicas que sostienen con la finalidad de poner en perspectiva la relación binacional tanto con el gobierno venezolano como con el brasilero a fin de determinar el impacto que la frontera tiene sobre su sociedad como un todo.

\section{Ubicación}

LosYe'kwana son un grupo de filiación lingüística caribe que vive a ambos lados de la frontera entre Venezuela y Brasil en las cuencas de los ríos Orinoco, Ventuari, Padamo, Caura, Paragua, Cuntinamo y Uraricoera (Silva Monterrey 2007, 2010 y 2015). En Venezuela se encuentran ubicados al sur del país en dos Estados diferentes: El estado Amazonas y el estado Bolívar. Las comunidades ye'kwana venezolanas que están en la frontera con Brasil habitan en el estado Bolívar en la región de cabeceras del río Caura (Medewadi).

La cuenca del Caura tiene una superficie que constituye el 5\% del territorio venezolano con una longitud de aproximadamente $580 \mathrm{~km}$ recorridos por el río Caura que es el segundo afluente de importancia del Orinoco en su margen derecha (Vargas y Rangel, 1996: 48-53) (Ver Mapa 1). 


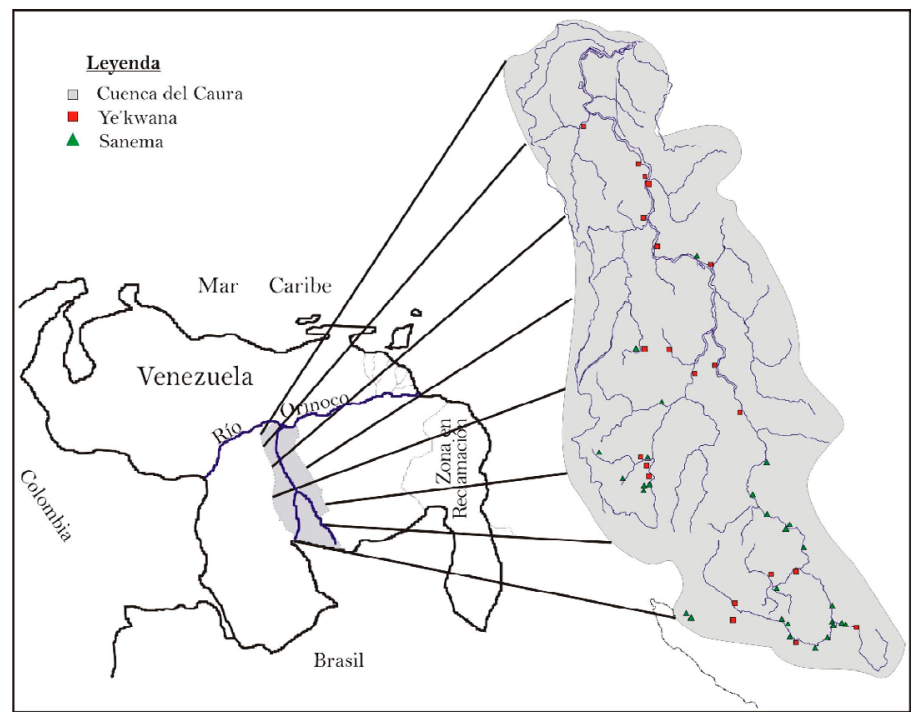

Mapa 1. Ubicación de las comunidades ye'kwana y sanema en la cuenca del Caura en Venezuela

Esta región posee suelos forestales de gran fragilidad y está cubierta en un 90\% por diferentes tipos de bosques de tierra firme (Fuentes y Madero, 1996: 44-47; Marin y Chaviel, 1996: 60-65). La misma se mantenía prístina hasta el año 2006 cuando una invasión minera inició su proceso de destrucción (Silva Monterrey y Núñez, 2006).

En la parte alta de la cuenca del Caura, en las cabeceras, habitan mayoritariamente los Ye'kwana junto con los Sanema (sub-grupo yanomami) y los Hoti de forma intermitente (Silva Monterrey, 2006). En la parte baja, al norte, viven los Pemón en poblados mixtos, además de los Kari' na y poblaciones mestizas no indígenas (criollas). La población ye'kwana total en Venezuela es de 7.997 personas de los cuales 3.280 viven en el estado Bolívar mayoritariamente en la cuenca del Caura que concentra al 34\% de los poblados ye'kwana del país asentados en alrededor de veinte poblados (RBV, 2011). Mientras que en Brasil los Ye'kwana se ubican en cuatro comunidades con una población total de 593 personas (População Indígena no Brasil, 2017).

Cabe decir que la zona fronteriza del Caura por el lado venezolano es de difícil acceso dado que este es eminentemente fluvial siendo el Caura un río caudaloso interrumpido por caídas de agua y raudales; un viaje por río en embarcación con motor fuera de borda desde la población de Maripa (que es una de los primeros pueblos no indígenas en la cuenca baja del Caura) puede durar hasta una semana 
y media remontando el río hasta Kanarakuni que es la población ye’kwana más grande que se encuentra cerca de la frontera (aproximadamente a $30 \mathrm{~km}$ ). También hay comunicación aérea por lo que existen en algunos poblados pistas para el aterrizaje de pequeños aviones; aunque la cantidad de bienes y servicios que reciben estos asentamientos fronterizos de la sociedad venezolana son bastante limitados. Desde Ciudad Bolívar, la capital del estado, hasta Kanarakuni son alrededor de tres horas en avioneta. El transporte aéreo a esa zona desde Venezuela es muy limitado (por lo riesgoso y costoso) y se hace solo en ocasión de viajes especiales de turistas a Kanarakuni o en el marco de operativos del Estado ya sea por cuestiones militares o para solventar alguna situación de salud, lo cual es poco frecuente.

Consideramos que dada la cercanía geográfica, desde las comunidades ye'kwana brasileras se llega a las poblaciones urbanas no indígenas de ese país más fácilmente que a las venezolanas criollas que distan a más de $500 \mathrm{~km}$ lineales por río hacia el norte. Los grandes viajes son emprendidos sobre todo por los hombres quienes son afamados y diestros en el arte de la navegación, razón por la cual en algunas comunidades fronterizas no es raro encontrar poblados con pocas personas, mayoritariamente mujeres.

Las comunidades fronterizas son bastante tradicionales conservando del lado venezolano el sistema constructivo de una sola casa redonda u oblonga con divisiones internas (Silva Monterrey, 1992); tenemos referencias que del lado brasilero las casas son de madera y que además de una pista de aterrizaje hay una oficina de la FUNAI y un puesto militar. Debido a la lejanía, la presencia de las instituciones gubernamentales venezolanas es precaria en esas comunidades lo que propicia el intercambio de los Ye'kwana con las instituciones de Brasil en búsqueda de asistencia, particularmente sanitaria, como veremos más adelante.

\section{Un solo pueblo de origen diverso}

La autodenominación del grupo es Ye'kwana pero en la literatura han sido reportados con al menos 54 nombres diferentes entre los que se destacan Dekuana, Ihuduana, Kunuhana, Mayongong (con siete variaciones), Pawana y Makiritare entre otros (Silva Monterrey, 2015: 89).

Los Ye'kwana del río Parime, bajo el etnónimo de Guadungamos, son mencionados por los colonizadores europeos en 1758 durante la Expedición de Límites oficializándose el contacto directo con los “Makiritare” en el río Padamo en 1759 (Bisbal y de De la Fuente en Michelena y Rojas, [1867] 1967: 163, 172); en esa 
época una de las actividades preponderantes que se desarrollaban en toda la región era la actividad esclavista la cual tuvo un gran impacto sobre las poblaciones indígenas que se vieron severamente diezmadas debido a ella (Silva Monterrey, 2015: 67-70). Los Ye'kwana consideran que el esclavismo es negativo y rechazan su práctica, sin embargo, hay indicios de que algunos de los grupos con los que se mestizaron lo practicaron.

Cuando Humboldt pasa por las cabeceras del Caura a finales del siglo XVIII y principios del XIX dice que todas esas regiones estaban desoladas, sin embargo, había grupos que habían sobrevivido como el Makiritare (Humboldt, [1804] 1991 IV: 61, 142). El mismo panorama de desolación que observa Humboldt pero producto de las enfermedades es observado por Koch Grünberg en 1911 ([1917] 1979 I: 236).

A finales del siglo XIX y primeras décadas del siglo XX se produjo el boom cauchero, periodo en el que los Ye'kwana sufrieron persecución, atropellos y relaciones laborales prácticamente como las de esclavitud con consecuencias similares a las del siglo XVIII con disminución poblacional, dispersión y movilización constante como mecanismos de supervivencia. Durante estos dos procesos de corte esclavista numerosos pueblos indígenas desaparecieron. Los Ye'kwana fueron los sobrevivientes de esta debacle demográfica y herederos de la tradición cultural de muchas etnias que vivían en la región.

Los Ye'kwana se reconocen como un solo pueblo, sin embargo, su identidad se nutrió de la diversidad cultural de pueblos hoy desaparecidos (Silva Monterrey, 2013). La cuna de los Ye'kwana se ubica en el alto Orinoco y el Cunucunuma; famosos por su habilidad como marineros y comerciantes son un pueblo acostumbrado a entrar en contacto con otras poblaciones, indígenas y no indígenas, y a desplazarse en un amplio territorio. Los Ye'kwana con estas dos cualidades para sobrevivir se mestizaron con otros grupos indígenas y se expandieron incorporando las identidades y las culturas de esos grupos a su propio bagaje cultural predominando la identidad ye' kwana pero quedando como un indicio de esa identidad constitutiva la diversidad de etnónimos, el conocimiento de los toponímicos, y numerosas historias en las que de manera secreta se reconoce la diversidad de base.

En el caso de los pueblos que se encuentran en la frontera con Brasil, nosotros sabemos de acuerdo a Kock Grünberg ([1917] 1979 I: 289) que los grupos que aparentemente vivían en la zona de las cabeceras de los ríos fronterizos con Brasil eran de filiación lingüística arawak. Estos grupos arawak son absorbidos por la expansión 
ye'kwana que viene del río Matacuni desde el noroeste y que se detiene en el Auaris cuando encuentra la presencia yanomami.

A pesar de la diversidad de base, los Ye'kwana tienen un cuerpo de mitos que les unifica como pueblo y en los cuales se da cuenta de su origen, de sus valores, de su territorio y de sus fronteras. En algunos de estos mitos se incluye a parte del territorio brasilero. Un ejemplo de ellos es el relato, en la versión del Caura, del recorrido mítico de Kuyujani quien demarcó el territorio ye'kwana en el cual se incluye al Parime y Auaris (Silva Monterrey, 2015: 252). (Ver Mapa 2).

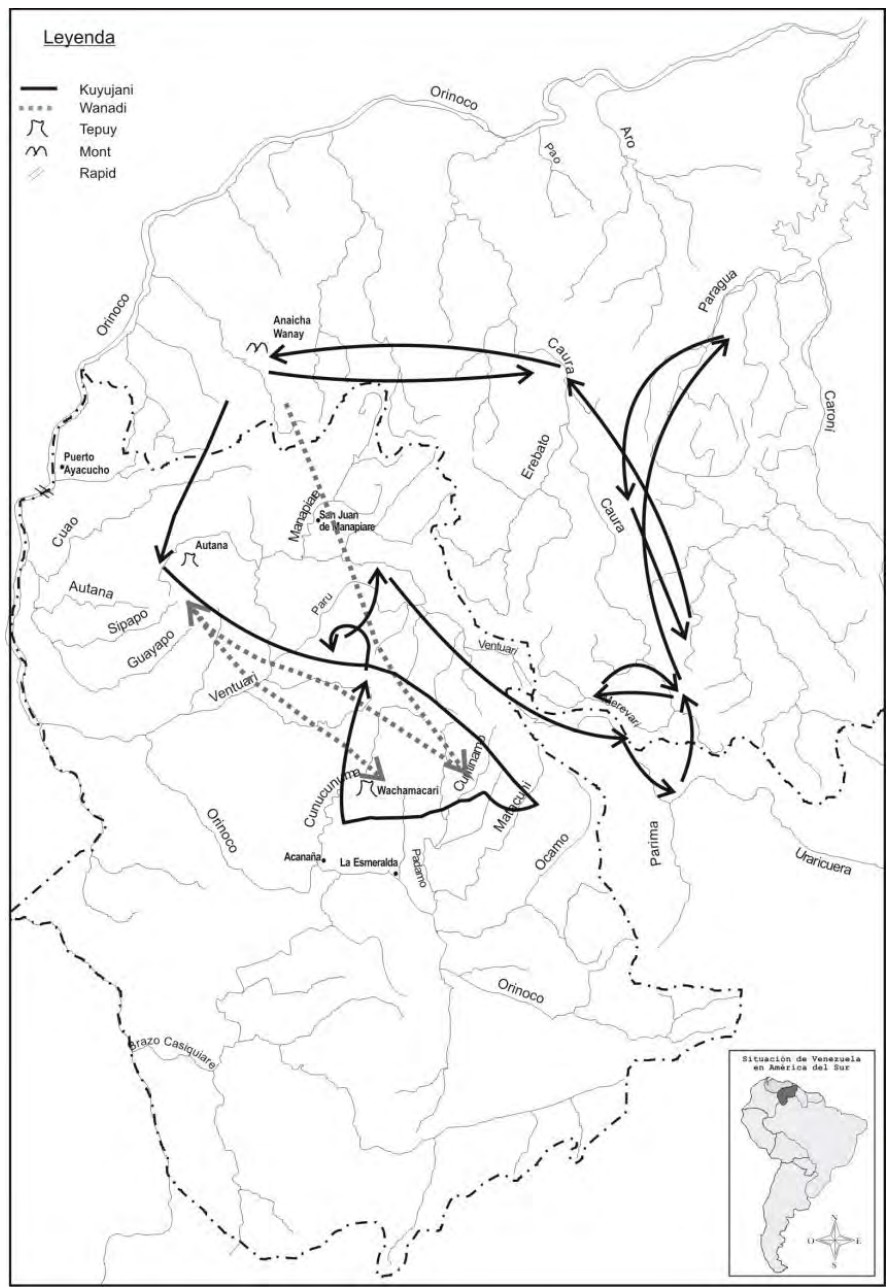

Mapa 2. Recorrido de Kuyujani durante la demarcación del territorio ye'kwana. Versión del Caura narrada por Napoleón Navaja. 
Por otra parte, y solo para mencionar otro relato que incluye a los Ye'kwana brasileros en los mitos, referiremos la historia de Sicha Muna y Yureke en la cual se señala que estos dos héroes míticos fueron a Kanarakuni y Farimiña (la Sierra Parime en Brasil), estuvieron recorriendo esa zona y luego migraron a otras regiones del país y del mundo.

Un fragmento de ese relato de la tradición oral narrado por Napoleón Navaja dice:

"El primero en hacer un Wejunma fue Wanadi y luego Yureke y Sicha Muna. Sicha Muna se crió en Cacurí y cuando estuvo grande, hicieron una churuata que está en Cacurí y que ahora se ve como un cerro y que se llama Öttöümö jüdü, eso lo hizo con su hermano Yureke.

Entonces Sicha Muna se fue con su hermano a Kanarakuni, allí hicieron el pueblo de Kanarakuni, pero Yureke se marchó a Farimiña (Parime, Brasil), allí hicieron también una churuata que fue construida por Yureke. Sicha Muna se quedó en Kanarakuni, el nombre de la churuata de Farimiña era Watajüdü. Luego Yureke se fue a Caracas y Sicha Muna se quedó en Kanarakuni y se casó. Su suegro se llamaba Wikara. Mientras, Yureke estaba solo en Caracas decidió buscar a su hermano en Kanarakuni. Entonces se juntó con su hermano Sicha Muna en Kanarakuni yYureke le dijo vámonos para Caracas, yo no te quiero dejar a tí aquí. Sicha Muna le respondió que él no quería ir para allá, entonces Yureke le dijo, está bien.

Yureke cargaba Wejunma que Wanadi, su papá, le había dado. Wejunma es una planta; Yureke cargaba bastante de esa planta, cargaba además un pito y un caracol. Yureke estaba una noche durmiendo en Kanarakuni y quiso irse a Caracas y dejó a su hermano tranquilo. Se fue con su chinchorro y su maletín y tocaba el caracol y el pito.

Sicha Muna al darse cuenta de que su hermano se iba, se puso a llorar, habló con su esposa y su suegro y se fue con su hermano, y el suegro estuvo de acuerdo. Entonces Yureke se juntó con Sicha Muna abajo, cuando lo alcanzó le dijo:- mira hermano, yo voy contigo- y Yureke le respondió -bueno vámonos- . Así se fueron a Caracas, dejaron Kanarakuni y Fadimiña...” (Silva Monterrey, 2015: 347-348).

Pero los Ye'kwana de Brasil no solo ocupan un territorio que está dentro de la tradición del grupo, sino que además guardan proximidad social dialecto-parental dentro del área definida por nosotros como "Merewari” en el que predomina el dialecto ye'kwana-ijuduana (Silva Monterrey, 2015: 97, 128-130) (Figura 1). 


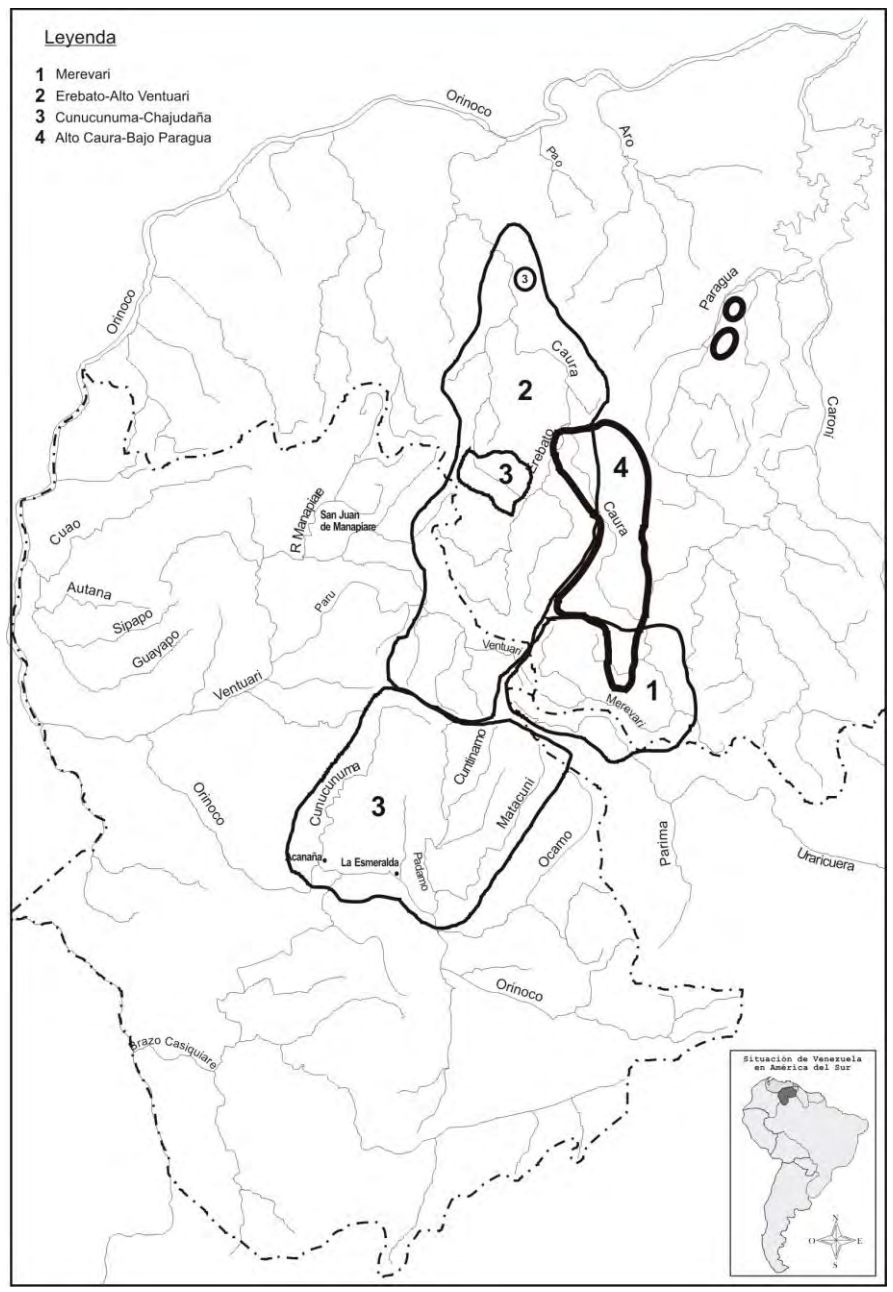

Figura 1. Proximidad Social dialecto-regional ye'kwana.

La gente que vive en las cabeceras del Caura y en Brasil baja por el río Caura y visita a las comunidades de Fedekuniña y Adawaña sobre todo, que son dos comunidades que hoy en día están haciendo mucha minería. Normalmente estos pueblos no pasan a través del Salto Pará, sino que lo hacen a través del rio Chanaro que es un afluente del Caura y se van hacia la zona del Paragua en donde hay dos pequeños poblados ye'kwana entrando así en contacto además con los Pemón quienes son un grupo que también vive en la frontera venezolana con Brasil (Ver Mapa 1).

Eso no quiere decir que ellos no visiten otros poblados, sino que esta es la ruta preferencial de visitas e intercambios.

Me tocó en una oportunidad estar en un poblado del río Erebato (afluente prin- 
cipal del Caura) en Jüwütünña, y ver que unos ye'kwana brasileros estaban de visita. En esa ocasión, uno de los muchachos brasileros se enfermó y murió; entonces como un mecanismo para mantener la paz interna y la tranquilidad en función de las creencias mágico-religiosas, se conformó una comisión que salió hacia el poblado brasilero, en un viaje que duró tres meses, a dar explicaciones sobre la muerte del muchacho y a asegurarles que no había ningún acto de brujería de por medio ni ningún tipo de hostilidad interna hacia el poblado ye'kwana brasilero. Es claro que la distancia geográfica genera entre los Ye'kwana cercanía afectiva y colaboración estrecha si se está cerca, y tensión o desconfianza si la distancia entre ellos es considerable. A menudo poblados contiguos son producto de escisiones internas de una misma comunidad tal y como ocurrió con Karanarakuni y Fedekuniña o Jüwütünña y Anadiqueña por solo dar un ejemplo. Nosotros determinamos que una de las características más resaltantes de la identidad como pueblo es la idea de su sociedad como un espacio de paz delimitado por las relaciones parentales. Se es ye'kwana en tanto se le pueda reconocer como pariente en un espacio de paz y armonía interna.

Vimos también como el otro señor brasilero que acompañaba al difunto se casó en el poblado anfitrión, de acuerdo a los cánones matrimoniales de la cultura ye'kwana de matrimonio preferencial entre primos cruzados bilaterales, lográndose retrasar a través de la genealogía los ancestros comunes y establecer los vínculos que les ligaban como parientes. Este hombre no regresó a Brasil quedándose en el pueblo e integrándose totalmente a la comunidad.

Entre los Ye'kwana son importantes las visitas no solo para participar en las fiestas (Silva Monterrey, 2016), aprender la historia, para comerciar o para ver a los parientes sino también para colaborar en los trabajos de las comunidades que así lo requieren, particularmente cuando se emprenden grandes construcciones, como la de hacer una nueva Öttö. ${ }^{1}$ Estas ocasiones son momentos de renovación de los nexos internos que a menudo terminan en matrimonios o promesas de visitas.

En un esfuerzo por mantener mayor contacto entre sí los Ye'kwana del Caura, que están vinculados por una red de radio-comunicación extensa a la cual se conectan todos los poblados de la cuenca para compartir noticias a horas determinadas, trataron de incorporar a los poblados ye'kwana brasileros a esa red de radio-comunicación encontrándose con el obstáculo de que tenían que pedir un permiso especial que era obligatorio y que se obtenía de las autoridades gubernamentales brasileras en Manaos. 


\section{Relaciones interétnicas}

\subsection{Relaciones con los Sanema y los Pemón}

Las relaciones interétnicas se dan tanto con los Sanema que están en las cabeceras como con los Pemón de la cuenca del Caroní también en la frontera con Brasil.

Desde tiempos inmemoriales los Ye'kwana estaban en guerra con los Sanema-Yanomami cuyas hostilidades se vieron relegadas a un segundo plano durante la época del caucho a principios del siglo XX. Pero hacia fines de los años 1950's los Ye'kwana vencen a losYanomami (Sanema) quienes permanecen en el Caura realizando trabajos para los Ye'kwana en poblados cercanos hasta los años 1990's cuando se funda la Organización Kuyujani y los Ye'kwana deciden acabar con la relación de inequidad que mantenían hasta entonces.

Los Ye'kwana y Sanema intercambian productos venezolanos dados por los Ye'kwana a cambio de fuerza de trabajo para las labores agrícolas o del hogar por parte de los Sanema. Se dan incluso algunos matrimonios entre hombres ye'kwana con mujeres sanema.

Con otros indígenas como los Pemón se mantuvo hasta mediados del siglo $\mathrm{XX}$ un comercio interétnico muy activo y los Ye'kwana intercambiaban rallos de yuca, embarcaciones y perros por ollas, hamacas y escopetas que daban los Pemón (Coppens 1981) siendo la ruta preferencial la vía fluvial de cabeceras del Caura y Uraricuera. Es decir que las comunidades ye'kwana del Auaris eran las primeras dentro de la red comercial pemón-ye'kwana al estar en el camino principal hacia la venezolana zona pemón y hacia Guyana.

Hoy sin embargo, este comercio se ha debilitado aunque se mantienen algunos contactos quizá más por el placer de encontrarse que por la necesidad misma de los productos. De esta manera los Ye'kwana siguen haciendo rallos de yuca y embarcaciones (curiaras) para comerciar por encargo con los Pemón quienes proporcionan chinchorros de algodón u ollas de barro. La casi desaparición del comercio interétnico tiene que ver con el hecho de que los productos tienden a ser sustituidos por bienes occidentales pues ahora hay máquinas de moler yuca (usadas en las comunidades que tienen plantas eléctricas) así como hamacas de nylon. Sin embargo, los Ye'kwana mantienen el comercio de embarcaciones como un producto importante tanto para el comercio con los Pemón como con los venezolanos no indígenas.

\subsection{Relaciones con los no indígenas (criollos)}

Los Ye'kwana de Venezuela tienen una larga historia de contacto con la sociedad 
nacional lo cual ha quedado plasmado en los mitos, entre ellos tenemos el Watunna (historia) en el que se hace referencia a la relación de comercio con los españoles a finales del siglo XVIII y entre estos se reconoce a Centurión (Centeriya) desdoblándose desde entonces el mito en el reconocimiento del español malo y el bueno encarnado por Centeriya (Silva Monterrey, 2015: 62). Durante ese mismo periodo también se habla en los mitos de Ta'jo (huella-el colonizador europeo-) quien andaba en las cabeceras del Caura cazando gente para esclavizarla; a Judunko (el extranjero, los holandeses) con quienes se comerciaba en las cabeceras del Caura y hacia la región actual de Guyana (Civrieux, 1970).

En la época del caucho, hasta 1921, la siniestra presencia de Funes marca una relación negativa de las comunidades con el resto de la sociedad nacional. Pero cuando acaba el periodo cauchero se revive la guerra con los Yanomami y los Ye'kwana se ven nuevamente obligados a huir hasta que les vencen como señalamos en el aparte anterior. Es decir que relaciones negativas o positivas se mantienen tanto con poblaciones indígenas como con no indígenas.

Hasta los años 1940's los poblados ye'kwana eran asentamientos pequeños como de 30 personas que vivían en una sola casa comunitaria y que se encontraban dispersos dentro del territorio como un mecanismo de supervivencia heredado de siglos de conflictos. Esa dinámica de movilización de los poblados, de dispersión, y fundación de nuevos asentamientos es propia de la cultura ye'kwana y tiene que ver no solo con los conflictos sino con la adaptación a los ecosistemas selváticos.

Sin embargo, a partir de los años 1950's, y con la presencia de los misioneros tanto católicos como evangélicos, se inicia un proceso de sedentarización con la finalidad de desarrollar programas de educación y asistencia sanitaria fundamentalmente. Este proceso hace que los poblados crezcan al punto de que tenemos poblados que ya tienen una antigüedad de 59 años como Jüwütünña, cuya población alcanza las 200 personas, lo cual es un fenómeno novedoso para este tipo de sociedades. A pesar de la sedentarización la formación de nuevos poblados no se ha detenido y han nacido otros; se ha visto que los grandes poblados a menudo están medio vacíos pues hay una constante movilización de los ye'kwana generándose ahora relaciones y asentamientos en sitios no tradicionales fuera del territorio en poblados criollos como en Maripa (Bajo Caura), en Boa Vista e incluso en Ciudad Bolívar (capital del estado Bolívar) en donde hay asentamientos en barrios como Los Caribes y Hueco Lindo. En este último lugar losYe'kwana, con el apoyo de la misión católica, mantienen una casa adonde llegan los miembros del grupo para pernoctar 
cuando tienen que resolver asuntos en la Ciudad, de la misma manera que como lo hacen en la casa del mismo tipo de Boa Vista.

\subsubsection{Las misiones y el desarrollo comunitario}

El proceso de sedentarización ha sido propiciado por las misiones religiosas para facilitar la prestación de servicios de salud y educación. Las escuelas que ellos lograron instalar en las comunidades son escuelas públicas nacionales. Asimismo, promovieron la prestación de servicios de salud que dependen también del gobierno nacional pero son administradas por el gobierno regional logrando que se desarrolle una permanente formación de jóvenes ye’kwana tanto en el área de educación como de salud. Cabe señalar que las misiones católicas no realizaron conversiones ni bautismos lo que sí hicieron las misiones evangélicas Bautistas o de las Nuevas Tribus.

Con el desarrollo de los programas de educación y salud, se empiezan a generar diferencias en los Ye'kwana fronterizos pues ya no son escolarizados de la misma manera ni partícipes de programas de salud similares a ambos lados de la frontera. Es entonces a partir de los años 1960's cuando el Pueblo ye'kwana a ambos lados de la frontera empieza a sentir que existe realmente una frontera nacional puesto que son educados en idiomas diferentes, aprenden historias nacionales diferentes (unos la de la Brasil y otros la de Venezuela) e interactúan con sociedades de carácter y trato particular en cada caso. Es cierto que los Ye'kwana se sienten como un solo pueblo, pero se suma a la identidad propia un marcador de diferencia: la frontera que les divide en ye'kwana brasileros y venezolanos.

Las misiones ejercen también un papel importante en la economía propiciando nuevas formas de desarrollo económico a través de la formación de empresas como Tujuumoto en 1975 con fines agrícolas, de producción y comercialización de artesanías. Es necesario mencionar que fueron los misioneros católicos los que propiciaron la transformación de la artesanía tradicional en un producto solo para la comercialización con el desarrollo de aspectos estéticos para ser vendida en Ciudad Bolívar y en otras partes. Estas comunidades religiosas católicas promueven la creación de estos nuevos productos a la par de productos tradicionales como el café y el cacao (Coppens, 1981; Heinen, Velásquez y Tomedes, 1981).

Los Ye'kwana se van formando en los procesos administrativos y con su ya vasta experiencia comercial son exitosos con su empresa Tujuumoto formada inicialmente solo para Jüwütünña pero que luego va expandiéndose hasta constituirse en una empresa intercomunitaria. Ella sirvió de modelo para otras empresas interco- 
munitarias indígenas como UNYESA, cuyo centro era Sayu Shodinnña en el medio Erebato, e incluso otras empresas intercomunitarias que se crearon en el estado Amazonas como la de la UMAV en el Ventuari.

\subsubsection{El gobierno venezolano y sus programas}

La profesionalización de los Ye'kwana tanto en educación como en salud llevó a que ellos tuvieran mayor interacción con la sociedad nacional y que estos programas quedaran en manos de los propios ye'kwana al interior de las comunidades. Una de las consecuencias inmediatas fue que el Estado Nacional empezara a contratar a los Ye'kwana como funcionarios para atender esas tareas por lo que algunas personas de las comunidades empezaron a recibir dinero de forma regular como asalariados lo que tuvo incidencia en la economía de las comunidades. Este momento clave genera desequilibrios al interior de la sociedad ye'kwana generándose un pequeño núcleo de personas con dinero constante frente a otros que no lo tenían. Las comunidades exigen la creación y repartición de puestos de trabajo iniciándose para muchos un proceso de dependencia económica del Estado Nacional que además exige la implementación y desarrollo de las políticas nacionales. Se reciben en una comunidad varios sueldos: los de los maestros, de los microscopistas, de los encargados de malariología, de los enfermeros entre los más comunes, empezando entonces las comunidades a entrar en un proceso de salarización que no existía previamente. Cuando se introducen los salarios, el desarrollo económico que propiciaba la autonomía con las empresas estimuladas por las misiones religiosas se transforma ahora en procesos de dependencia de las instituciones nacionales.

Con esta aparición de los sueldos se inicia también un proceso clientelar y los partidos políticos empiezan a tener algún tipo de incidencia en las comunidades aunque pequeña en los años 80' y 1990's.

Para comprender esta última situación es necesario decir que los Ye'kwana en Venezuela se encuentran ubicados no solo en dos Estados diferentes sino también en Municipios diferentes. Los del estado Bolívar por ejemplo se encuentran en tres municipios que tienen jurisdicción en la región del Caura y del Paragua. Estas instancias municipales también generan empleos y promueven la lealtad política, incluso de forma directa mucho más que las instancias de carácter nacional. Los funcionarios municipales, a menudo contratados como promotores de programas para la alimentación, transporte, desarrollo económico y salud, así como asistencia social entre otros, para conservar su puesto de trabajo se convierten en activistas políti- 
cos que promueven la gestión del alcalde de turno y sus programas político-partidistas, lo que ha sido en algunos casos motivo de diferencias internas. Hoy en día los Ye'kwana del Caura son mayoritariamente afectos al gobierno nacional que está en la misma línea que el gobierno estatal y municipal tal y como lo demuestran los resultados electorales en estas comunidades (CNE, 2017).

\section{La educación básica}

En Venezuela en la Constitución Nacional de 1961 había un solo artículo, el No. 77, que refería que se establecía un régimen de excepción para los indígenas hasta tanto estos fueran incorporados a la vida de la nación (RDV 1961). En esa época la visión que se tenía es que los indígenas iban paulatinamente a desaparecer y que este era una suerte de campesino por lo que se hicieron en ese periodo muchos programas nacionales con una visión integracionista y agrarista entre los cuáles los cultivos ya mencionados a la par de introducción de ganado vacuno, iniciativa esta última que fracasó.

Esa visión se contradecía en la práctica con el desarrollo de un movimiento indigenista e indígena por lo que fueron apareciendo organizaciones indígenas que luchaban por la reivindicación de sus derechos. Los Ye'kwana participaron en este proceso y uno de sus miembros, Ye'kuana René Estaba, joven estudiante de medicina de la comunidad de Jüwütünña, fue muy activo en los mismos a nivel regional formando parte del Movimiento Indígena de Guayana (MIG) y luego propiciando la formación de una organización indígena nacional que hasta el día de hoy representa a todos los grupos indígenas del país denominada Consejo Nacional Indio de Venezuela (CONIVE).Ye'kuana René conjuntamente con un grupo de jóvenes inquietos en las comunidades del Erebato decidió dar el salto de constituir una organización interétnica en la Cuenca del Caura que agrupara a los Ye'kwana y Sanema con fines de defensa de sus derechos y reivindicación del territorio en los 1990's, la cual se denominó Organización Indígena de la Cuenca del Caura "Kuyujani”.

Fue gracias a la presión de estos movimientos indígenas y de la comunidad de aliados (antropólogos, sociólogos etcétera) que se impulsó la implementación del Decreto 283 de Educación Intercultural Bilingüe que había sido promulgado en 1979 (RDV, 1979) por lo que se inicia un proceso para tratar de estandarizar las lenguas indígenas y de promover la escritura y el aprendizaje en los idiomas indígenas a partir de la Dirección Nacional de Asuntos Indígenas (DAI); así como de producir materiales de lectura en el propio idioma con la finalidad de reforzar la 
identidad de cada Pueblo. Se hacen cambios en las escuelas primarias en las comunidades, se exige la escolarización hasta el tercer grado al menos en el propio idioma, al tiempo que se refuerza la identidad nacional a través de la enseñanza de la historia nacional aun cuando esto se hiciera en el propio idioma indígena. En el Caura esto no era una novedad pues los misioneros habían venido escolarizando en el idioma indígena, creando alfabetos y trabajando en la escritura de la lengua desde hacía muchos años. La particularidad estribaba en que los misioneros evangélicos escribían con "U” y "C", por ejemplo, envés de "W" o "K" como lo hacen los católicos. Los lingüistas sugieren que la ortografía debe ser la que usan los católicos, más sin embargo el gobierno en los textos educativos posteriores a la Constitución de 1999 (RBV, 2000) tratan de soslayar esta diferencia y proponen un texto en el cual muestran las palabras en los dos tipos de ortografía (MECD, 2002).

Antes de los 1980's los misioneros apoyaban la escolarización básica en las comunidades del Caura pero seleccionaban a algunos alumnos para que fueran a internados religiosos en el estado Amazonas, junto a indígenas de otras etnias, a continuar sus estudios de educación media. El gran cambio después de los 80's es que los niños son masivamente escolarizados dentro de las comunidades por maestros indígenas formados en los internados religiosos; son este grupo de jóvenes que salieron a escolarizarse en los 70's los que retornan a las comunidades para ser funcionarios y líderes en los diferentes programas de salud, educación y economía al frente de la empresa intercomunitaria o en la política indígena nacional como Ye’kuana René Estaba.

A partir de los 1990's se crea una Escuela Granja cerca de Jüwütünña pero eso no es suficiente y los padres de los jóvenes al concluir la educación primaria les envían a Maripa y Ciudad Bolívar (ya casi no al internado) a concluir los estudios de secundaria y eventualmente continuar los superiores. Los niños son escolarizados a partir del cuarto grado tanto en su propio idioma como en castellano, hay un conocimiento de la historia nacional, de los valores nacionales y el pensum es de carácter nacional aunque con un espacio para lo particular. Allí se acentúa el proceso de transculturización, los niños cantan el himno nacional y particularmente en las comunidades fronterizas esto se da cotidianamente. En Kanarakuni, a $30 \mathrm{~km}$ de Brasil, se notaba mayor énfasis en los aspectos ligados a la nacionalidad izándose la bandera al son del himno nacional todos los días, lo que no se observaba de la misma manera en otras comunidades.

Hacia el 2000 percibimos el inicio de un éxodo de jóvenes ye’kwana del Caura a las comunidades criollas, primero a Maripa y luego a Ciudad Bolívar. En un primer 
momento muchos jóvenes varones iban a Maripa a formarse, mientras que las muchachas seguían siendo enviadas a los internados en Amazonas para que continuaran también sus estudios de educación secundaria.

A lo largo de la primera década del siglo XXI las jóvenes empiezan también a estudiar en Maripa y Ciudad Bolívar, incluso solas, alojándose en casas de familia o con otros ye'kwana que empiezan a establecerse de forma casi permanente en las ciudades. Las consecuencias de esto las estamos viendo en la actualidad. En los últimos años nosotros hemos observado una gran cantidad de muchachas ye'kwana que fueron educadas en escuelas no indígenas; niñas que salieron de 12 o 13 años o antes de las comunidades; niñas que no guardan una vinculación estrecha con las comunidades como segunda generación que sale a estudiar fuera y que ahora tienen hijos y no les quieren hablar en el idioma ye'kwana.

Antes los estudiantes retornaban masivamente a las comunidades durante las vacaciones, ahora no se retorna ni siquiera en las vacaciones y muchos niños viven desde su nacimiento fuera del territorio en los poblados criollos. Las muchachas de la primera generación que estuvo en los internados salieron y regresaron, estas jóvenes de la segunda generación no tienen una vinculación estrecha con las comunidades, y poco a poco se va perdiendo la base comunitaria en un franco proceso de transculturización y de pérdida del idioma.

Se sigue manteniendo el sistema de matrimonio preferencial entre primos cruzados, el sistema dravidiano de matrimonio (Silva Monterrey, 2015); el cambio no es aún en la estructura matrimonial sino en el uso del idioma. Pues, como ya lo señalamos, tanto la madre como el padre de los niños hablan castellano, y no necesariamente usan el idioma ye'kwana de forma permanente entre ellos, y menos aún con los niños.

Las comunidades fronterizas, sin embargo, dada la gran distancia que les separa de las comunidades no indígenas venezolanas, lo difícil de la navegación y la escasez de recursos monetarios, viven este proceso de manera menos intensa que las comunidades del Erebato. Ellas siguen permaneciendo un poco aisladas del resto aun cuando las instituciones escolares hagan su trabajo de recordar que se encuentran de un determinado lado de la frontera.

\section{La educación universitaria}

Una vez concluida la educación secundaria muchos jóvenes intentan realizar estudios superiores sin embargo son pocos los que han logrado concluirlos con éxito. 
Uno de ellos, el ex-coordinador de la Organización Kuyujani, se graduó de abogado, estuvo un tiempo en la ciudad y luego regresó a su comunidad en Boca de Nichare sin que actualmente ejerza su profesión. Los más exitosos en la conclusión de estudios superiores han sido los maestros quienes han obtenido en la mayoría de los casos su título de licenciatura de Educación Intercultural Bilingüe en la Universidad Pedagógica Estatal Libertador (UPEL) que tiene un programa especial para ellos.

Muchos estudiantes han viajado a lugares distantes como Mérida a estudiar en la Universidad de los Andes licenciatura en Química o Estudios Internacionales; o en otras universidades Turismo o Informática pero la gran mayoría sin éxito. En el área de salud la formación que sigue prevaleciendo es la de nivel técnico medio como enfermeros y microscopistas, aunque recientemente un joven de Jüwütünña se graduó de Licenciado en Enfermería y otro, en una universidad no tan reconocida, de Médico Integral comunitario. Cabe señalar que algunas universidades venezolanas tienen programas especiales para dar cabida a los estudiantes indígenas y en el sistema nacional de ingreso a la educación superior el ser indígena facilita el proceso. Es decir que el Estado Nación tiene una política inclusiva en relación a los pueblos indígenas en materia de educación superior, más sin embargo la prosecución de estos estudios se ve entrabada por la situación económica de los estudiantes indígenas quienes no cuentan con recursos monetarios suficientes para mantenerse en las ciudades. El señor ye'kwana que se graduó de abogado lo hizo con el apoyo de una beca alemana.

Las comunidades tratan de apoyar a sus estudiantes, antes de una forma muy organizada las comunidades enviaban cada una provisiones alimenticias como mañoco o casabe, pero eso se ha perdido y hoy en día lo que se observa es que los jóvenes estudiantes tienen muchas carencias. La mayoría de los estudiantes proviene de las comunidades del Erebato y son raros los que vienen del Alto Caura o de las comunidades fronterizas a proseguir estudios superiores en Ciudad Bolívar. En este sentido las comunidades fronterizas se mantienen más reservadas en cuanto a sus expectativas de desarrollo.

\section{Instituciones científicas y Organizaciones No Gubernamentales (ONG)}

Desde finales de los años 1980's las instituciones académicas han hecho presencia en las comunidades fronterizas, particularmente en Kanarakuni en donde la Universidad de Oriente tenía una Estación de Investigación en la cual los científicos en el área de medicina desarrollaban trabajos de investigación sobre las enfermeda- 
des tropicales dando como resultado publicaciones sobre las endemias propias de la región. La visita de los científicos era esporádica, así como la de exploradores y biólogos entre los que destaca Charles Brewer Carías quien visita la zona con fines de estudio de las oquedades en la sima del complejo tepuyano “Jawa Sarisariñama”.

En los 1990's realizan estudios científicos en toda la región profesionales contratados por la compañía de Electrificación del Caroní "EDELCA" para determinar la factibilidad de desvío del río Caura al río Paragua. La mayoría de estos estudios, en cuanto a la caracterización de la zona, fueron recogidos en dos volúmenes de Scientia Guianae No. 6 y No. 7 (HuberY Rosales, 1996, 1997).

Afortunadamente el proyecto gubernamental fue desechado pero significó un gran peligro para las comunidades de la zona que, de haberse efectuado el proyecto, hubieran quedado incomunicadas las de la frontera o sumergidas en el agua las del curso medio del Caura, además del desecamiento del río Caura desde Chanaro en varios cientos de kilómetros.

Las comunidades ye'kwana fueron muy activas en relación al rechazo frente a este proyecto y establecieron una red de alianzas nacionales e internacionales que contribuyeron a que el proyecto de trasvase no se realizara. Adicionalmente se organizaron para la defensa del territorio en la Organización Kuyujani y contactaron a ONG internacionales para realizar la demarcación de sus tierras.

Como resultado, y con el financiamiento del World Rainforest Movement (WRM), a través del Forest Peoples Programme (FPP) de Inglaterra, además del apoyo técnico de esa institución y de la Universidad Nacional Experimental de Guayana (UNEG) se desarrolló este proyecto que se concluyó exitosamente aun cuando hasta el día de hoy no se ha obtenido el reconocimiento de los títulos colectivos sobre la tierra (Colchester, Silva Monterrey y Tomedes, 2004).

Este proceso de demarcación significó un esfuerzo organizativo enorme y puso en conjunción a todas las comunidades de la cuenca del Caura para realizarlo, incluyendo a las poblaciones más apartadas. La demarcación además sirvió para revitalizar los nexos intraétnicos, recordar a los ancestros y a los mitos.

En esta misma época la Universidad Nacional Experimental de Guayana inició junto con la Fundación para el Desarrollo de la Ciencia y la Tecnología de la Región Guayana (FUNDACITE Guayana) un programa denominado Bioguayana uno de cuyos sub-programas era el de la Cuenca del Río Caura. Un hito importante fue la firma de un convenio de cooperación interinstitucional entre las comunidades del Caura representadas por la Organización Kuyujani, la UNEG y FUNDACITE 
Guayana para el desarrollo de Bioguayana-Caura en 1997.

Fruto de ese trabajo de cooperación interinstitucional incluyendo al FPP fueron los Programas de Formación de Parabiólogos ye’kwana y sanema, la creación de la estación científica "Ka'kada” y numerosas publicaciones entre ellas las "Pautas para el desarrollo del Plan de Manejo de los Hábitat Ye’kwana y Sanema de la Cuenca del Caura" (Silva Monterrey, 2012) entre otras.

Finalizando el milenio la ONG "Asociación para la Conservación de las Areas Naturales” (ACOANA) inicia un estudio para determinar la Vocación de la Cuenca del Caura en el que participaron no solo las instituciones antes mencionadas y los Ye'kwana a través de Kuyujani, sino otras ONG con interés en la conservación. Este trabajo no se concluyó, pues paulatinamente los recursos de las ONG se vieron limitados y sobre todo porque después del año 2004-2005, el Estado venezolano ha tenido una política contra las organizaciones no gubernamentales tanto las nacionales como extranjeras, lo que ha hecho que las ONG se hayan ido retirando paulatinamente de la cuenca del Caura quedando hoy en día solo ACOANA con un programa de salud para control de la malaria que tiene una presencia importante en las cabeceras del Caura y sobre todo en Kanarakuni. Es decir que tanto las ONG como las instituciones científicas se han prácticamente retirado de la zona y solo restan los servicios gubernamentales de educación y salud básicos.

\subsubsection{Gobierno Nacional y control del territorio}

Desde 2003 el Gobierno Nacional frente al reclamo territorial ye'kwana-sanema en el Caura realizó una serie de consultas y estudios para reglamentar el uso de las Areas Bajo Régimen de Administración Especial (ABRAE) que existen en la cuenca del Caura, y determinar cuál era la figura político-administrativa más adecuada en términos de reconocimiento de los derechos indígenas, de los pobladores no indígenas del bajo Caura y de la sustentabilidad de la misma. La proposición del Ministerio del Ambiente concluyó en un documento consensuado entre los diferentes actores de la Cuenca y con las ONG e instituciones académicas con actividades en la región. Esta propuesta reconocía parcialmente los derechos de los Ye'kwana y Sanema sobre el Caura a través del establecimiento de una zona indígena multiuso. Lamentablemente esa propuesta no fue decretada, debido, por un lado, a una invasión minera, y por el otro, a los recursos legales interpuestos por un grupo de ambientalistas ajenos a la región que con fines de proselitismo político proponían la creación de un Parque Nacional para el Caura. 
La invasión minera del año 2006 a la cuenca del Caura fue masiva y ya no se trataba solamente de la minería intermitente iniciada por los Ye'kwana y los garimpeiros brasileros desde hacía años, sino que en esta ocasión se trató de una invasión de miles de mineros venezolanos no indígenas entre los cuales había también mineros colombianos, guyaneses y brasileros (Silva y Núñez, 2006).

Este evento ha devastado de manera severa los ecosistemas del brazo derecho del río Caura en la zona del ríoYuruani; y la comunidad de Adawaña, que era una comunidad con constantes visitas de las diferentes comunidades indígenas del Caura y de Brasil, se convirtió en una comunidad minera que les acoge para esa actividad. En estas comunidades mineras se ha abandonado la economía tradicional y se ha dejado de sembrar, por lo que aquellos que aún lo hacen venden alimentos, lo que no sucedía antes, y se deja de lado el compartir característico de la sociedad ye'kwana.

Es paradójico que el Estado venezolano que propició en 1999 un cambio constitucional con el debido reconocimiento de los derechos de los Pueblos Indígenas a su identidad, medicina, organización social, educación y territorio (RBV, 1999), no reconozca por un lado los derechos de los pueblos indígenas sobre la tierra en esa zona pero tampoco sea capaz de controlar la presencia de los mineros y su actividad depredadora. Antes bien se establece un puesto militar en el Salto Pará el cual ha sido fuente de corrupción y desmanes en relación a los indígenas al punto de que en una ocasión uno de los militares sostenía relaciones de dominación sobre los Sanema a quienes obligaba a trabajar con poca remuneración en jornadas desgastantes casi esclavistas (González, 2014). Los militares que desde entonces están en el Salto Pará, supuestamente para resguardar el territorio, aún permanecen allí sin haber logrado un control efectivo de la zona, teóricamente protegida por cinco ABRAE, lo cual ha traído consecuencias graves para la salud, por la contaminación mercurial, a todos los habitantes de la cuenca, lo cual amenaza su supervivencia a mediano plazo (Red Ara, 2013; Watson, 2016).

La única respuesta del Estado frente al ecocidio del medio Caura y la creciente contaminación fue entonces el decreto de la zona como Parque Nacional (RBV, 2017), ABRAE que se sobrepone a las que ya existían sobre ese mismo espacio, sin ningún tipo de consulta a los Ye'kwana o los Sanema. Hasta el momento actual el decreto de parque no ha sido operativizado, sin embargo, constituye una imposición de nuevas normas administrativas sobre los habitantes de la cuenca. Hoy en día los Ye'kwana tratan de dialogar con el gobierno sobre este decreto para clarificar su situación en relación al mismo utilizando como herramienta de legitimación 
el mapa producto de la demarcación y el reclamo territorial realizado a principio del segundo milenio.

Adicionalmente el Estado venezolano, en una política depredadora como nunca antes se ha visto (ANRBV, 2016), empieza a desarrollar programas extractivistas como el denominado Arco Minero del Orinoco (AMO) (RBV, 2016). Este macroproyecto afecta parte de la contigua zona pemón que hace frontera con Brasil y la parte baja del Caura, además de todo el curso del Orinoco en su parte media y las regiones aledañas; el fin es la explotación de los diferentes recursos minerales que allí se encuentran. Para mitigar el reclamo de los indígenas, el gobierno decreta su incorporación al denominado AMO (RBV, 2016a). Este proyecto dada su magnitud tendrá un impacto indirecto en toda la cuenca del Orinoco incluyendo a Colombia.

Es claro que el gobierno venezolano no ejerce ni el resguardo, ni el control adecuado de su territorio en esta frontera la cual está prácticamente desguarnecida. Su presencia en el Salto Para ha sido utilizada para beneficio personal de los militares quienes realizan operativos intermitentes a la mina que no se cristalizan en un control de la actividad.

\section{Los Consejos Comunales}

El gobierno inicia hacia el año 2009 la organización de la población civil en Consejos Comunales (RBV, 2009). Estos consejos comunales tratan de imponerse como estructuras de promoción, ejecución y seguimiento de obras públicas en todos los poblados del país independientemente de su dimensión. La idea era que ellos administraran los servicios de agua, de electricidad, basura etcétera, que las comunidades no poseen, pero también otro tipo de programas que tienen que ver con la distribución de alimentos y programas sociales. La idea del gobierno era que estas organizaciones sustituyeran a mediano plazo a las alcaldías por lo que un conjunto de Consejos Comunales se agrupa en Comunas y estas en una Asamblea de Comunas al estilo de los Soviets de la antigua Unión Soviética.

Estas estructuras también llegan a las comunidades indígenas, entre ellas las ye'kwana. Las empresas intercomunitarias han desaparecido desde los años 90's y solo permanece activa en este momento la organización Kuyujani la cual respeta la organización social tradicional nutriéndose de ella.

El gobierno de Chávez, de corte populista, generó un sistema clientelar que deslumbró a muchos. Los indígenas en general y los Ye'kwana también apoyaron ese gobierno y en el proceso perdieron una buena parte de su autonomía. Los Conse- 
jos Comunales se instalaron en las comunidades y en algunas de ellas cada familia formó un Consejo Comunal para recibir los recursos del Estado. Esa situación hizo que se diera la espalda a la estructura tradicional, no se le hacía caso ni al Jefe ni al Concejo de Ancianos, cada familia resguardaba sus propios intereses monetarios debilitándose así la estructura organizativa propia. No había interés en el bienestar colectivo. Se temía que tomar decisiones dentro de la estructura política tradicional llevaría a compartir el dinero y a invertir en la comunidad. Es decir, se exacerbó el individualismo y se ignoró a las autoridades tradicionales en cada comunidad.

La organización Kuyujani también se debilitó en parte por la falta de recursos para sostenerse, pues las comunidades ya no quieren aportar tampoco para su sostenimiento, y las ONG que colaboraban con ella a través de la ejecución de proyectos conjuntos disminuyen, como ya dijimos, sus actividades en el Caura. El boom de los Consejos Comunales dura unos cinco años, luego empiezan a debilitarse porque el gobierno se encuentra en todo el país con una corrupción generalizada en el manejo de los recursos entregados a los Consejos Comunales y porque prácticamente no se les dan más recursos a estas organizaciones que son sustituidas por otras organizaciones chavistas. Hoy en día los Consejos Comunales sirven para viabilizar la distribución de alimentos con las denominadas bolsas CLAP y para la movilización político partidista del gobierno actual de Nicolás Maduro.

La minería divide a las comunidades tanto como los Consejos Comunales, algunos quieren que la actividad se mantenga otros la rechazan; pero a la par de la invasión de la zona por población foránea, el debilitamiento de los Consejos Comunales, los problemas de salud y ahora la declaratoria de Parque Nacional hacen que los Ye'kwana del Caura vuelvan sus ojos a sus jefes tradicionales y a la organización Kuyujani que se revitalizan.

La mala administración gubernamental ocasiona el deterioro económico y social del país que después de una bonanza sin precedentes, disminuye la inversión social; se produce la caída de todos los indicadores económicos, se inicia un proceso hiperinflacionario por lo que en este momento hay en Venezuela un franco retroceso de las condiciones de vida particularmente de salud y nutrición (Caritas Venezuela, 2017), que también impacta a las comunidades indígenas.

Muchas personas en las ciudades no tienen alimento, no tienen salario o este no les alcanza para satisfacer sus necesidades básicas. Los indígenas urbanos sufren las mismas penurias que la sociedad nacional por lo que los Ye'kwana que habían migrado a las ciudades o vivían allí por largas temporadas regresan a sus comuni- 
dades porque al menos en ellas producen sus propios alimentos. Sin embargo, la minería ha trastocado la vida en los poblados indígenas que en ella se han involucrado más activamente, no tanto en las comunidades fronterizas en donde la actividad es limitada.

El aspecto más delicado en esta situación es el referido a la salud y la alimentación. Para los Ye'kwana el primer aspecto es fundamental al encontrarse en una región endémica de paludismo. Pero dado que los programas de salud son administrados por el Estado, y es este el que proporciona las medicinas especiales como las del paludismo, que estas escasean; y no se pueden atender las necesidades de las comunidades, sin hablar de situaciones más graves como las enfermedades crónicas. Las comunidades Ye'kwana sufren también la crisis humanitaria nacional.

Frente a esto, los Ye'kwana como sociedad vuelven sus ojos a sus parientes del lado brasilero quienes les auxilian particularmente en el área de salud. Los Ye'kwana venezolanos llegan a Boa Vista en búsqueda de esos servicios después de haber intentado resolver la situación con las instituciones venezolanas. Tenemos el caso de un joven de Kanarakuni que llegó a Maripa y luego a Ciudad Bolívar (capital) y al no recibir la asistencia adecuada, se fue vía terrestre a Santa Elena de Uarién hasta llegar a la frontera con Brasil y con el apoyo pemón, consiguió el transporte en ambulancia para trasladarse a Boa Vista en donde en el hospital con su cédula venezolana y constatándose que era ye'kwana recibió un trato que le resultó satisfactorio. Los Ye'kwana comparan la atención médica que reciben tanto en Venezuela como en Brasil y dicen que en Brasil les dan a los enfermos y sus familiares alimentos, que hay orden en el sitio en donde son acogidos lo que no se percibe en la asistencia sanitaria en Venezuela. La gran diferencia no es solo el acceso a los medicamentos sino el que sea atendido no solo el enfermo sino también los familiares que en Venezuela pasan mucho trabajo sin recursos monetarios. En este caso el enfermo se fue vía terrestre a Brasil, pero no es raro que lo hagan también por río hasta el Auaris y allí se tomen un avión que les lleve a Boa Vista.

En este sentido nosotros podemos decir que los indígenas ye'kwana se benefician de su carácter de pueblo fronterizo. Por un lado, los Ye'kwana brasileros bajan a la mina en el medio Caura, se alojan y reciben apoyo de sus parientes, y por otro los venezolanos acuden a los servicios de salud del lado brasilero.

\section{La frontera entre Venezuela y Brasil}

Los Ye'kwana dicen que saben que la frontera existe para el país pero no para 
ellos como pueblo que mantiene una identidad única independientemente de la línea fronteriza. Como indígenas que están a ambos lados de la frontera entre Venezuela y Brasil, disfrutan de algunos beneficios que no tienen otros indígenas que nos están en esa frontera. Ellos señalan que dado que en Venezuela hay un deterioro enorme de la situación de salud ellos acceden a los servicios de salud brasileros beneficiándose de esos servicios porque ellos son ye'kwana.

He tenido información de que comunidades ye'kwana enteras, no solo de las zonas fronterizas sino de regiones alejadas de esta, están pensando seriamente en mudarse al otro lado de la frontera pero dentro del mismo territorio ye'kwana. Recientemente, hubo una asamblea ye'kwana en Juduwadunña (Brasil) en la que participaron los representantes de la Organización Kuyujani siendo uno de los temas más importantes el apoyo para colaborar en la resolución de la crisis de salud entre los Ye'kwana venezolanos.

Es claro que los Ye'kwana se unen frente a las adversidades y es en ese momento en donde se refuerza su unidad como pueblo. Un ejemplo claro es la situación actual y la crisis de salud a la que hemos hecho referencia. Esto solo es posible porque, independientemente de la frontera, las relaciones intraétnicas se mantienen.

\section{Conclusión}

Los Ye'kwana independientemente de la línea fronteriza, mantienen sus vínculos de parentesco e intercambio matrimonial reconociéndose como descendientes de los mismos ancestros y con rasgos culturales comunes. Entre los elementos culturales compartidos está la lengua ye'kwana, la visión del mundo dada por los valores y una propia historia común plasmada en los mitos y en la zaga de los seres míticos. Causa, por lo tanto, preocupación el desuso de la lengua entre los jóvenes ye'kwana venezolanos que empiezan a hablar exclusivamente en castellano a sus descendientes; si no se cuida este aspecto la identidad grupal a ambos lados de la frontera puede verse bastante afectada por el valor que tiene la lengua como medio de comunicación y sobre todo como vehículo identitario.

Este pueblo mantiene los vínculos internos reuniéndose, colaborando entre ellos y visitándose independientemente de la línea fronteriza de los Estados Nacionales que les dividen. Esa frontera Venezuela-Brasil no coincide con la determinada por sus héroes míticos como Kuyujani, las fronteras de su territorio tradicional tienen otros límites por lo tanto para ellos es solo una referencia más y no un obstáculo.

Sin embargo, ellos no son impermeables a las políticas de los Estados Nación 
envolventes. Cuando uno examina el impacto que las políticas del Estado venezolano han tenido y tiene en este momento en la sociedad ye'kwana puede pensar que ella está seriamente herida a consecuencia de esas políticas.

Enfrentar desde la organización social y valores propios la política errática del Estado venezolano de estos últimos años, en las que por un lado se les reconocen sus derechos pero por otro lado o no se les materializan o se generan programas que atentan contra la organización propia afectándoles negativamente, es lo que nos obliga a pensar que solo una visión clara y unificada desde el grupo es lo que les puede garantizar su supervivencia. En este sentido el objetivo fundamental de los Ye'kwana como sociedad, plasmado en sus mitos e historia, es y ha sido la preservación de su vida e identidad cultural como pueblo que trata de mantenerse diferenciado frente a dos Estados Nacionales, aunque no aislado sino más bien integrados y aprovechando los beneficios que ambos pueden proporcionarles.

Recebido: 01/12/2017

Aprovado: 15/02/2018

Nalúa Rosa Silva Monterrey, venezuelana, $\mathrm{PhD}$ em Antropologia Social e Etnologia pela Escola de Estudos Avançados em Ciências Sociais de Paris, leciona na Universidade Nacional Experimental da Guiana e coordena o Centro de Investigações Antropológicas da Guiana. Trabalha com os Ye'kwana da Bacia do Rio Caura, na Venezuela, desde 1986, focalizando sua organização social e política. Desde 1996, vem trabalhando na equipe do Programa Povos da Floresta (Reino Unido) com comunidades indígenas para a demarcação de seus territórios. Conta com mais de 50 publicações sobre os povos indígenas da Guiana venezuelana. Contato: nalua. silva@gmail.com

\section{Notas}

1. Öttö es la vivienda redonda comunitaria que se ubica en el centro de los poblados ye'kwana con paredes de bahareque y techo de palma. 


\section{Referencias bibliográficas}

ASAMBLEA NACIONAL de la REPUBLICA BOLIVARIANA de VENEZUELA (ANRBV). 2016. Informe de la Comisión Mixta a cargo evaluar el impacto ambiental y económico que ocasiona la explotación de la zona de desarrollo estratégico "Arco Minero del Orinoco". Caracas. (inédito).

CARITAS VENEZUELA. 2017. Disponible en: <http://revistazeta.net/2017/11/17/informe-caritas-venezuela-ante-la-onu/>.

CIVRIEUX, MARC de. 1970. Watunna. Mitología Makiritare, Caracas: Monte Avila Editores.

COLCHESTER, Marcus; SILVA MONTERREY, Nalua; TOMEDES, Ramon. 2004. Fomentando el Uso Consuetudinario de los Recursos Biológicos en el Alto Caura,Venezuela. FPP. Reino Unido. 70 pp.

CONSEJO NACIONAL ELECTORAL (CNE). 2017. Resultados parciales de los comicios regionales del Estado Bolívar (inédito).

COPPENS, Walter. 1971. Las relaciones comerciales de los yecuana del Caura-Paragua. Antropológica, 30: 28-59.

FUENTES, Jose y MADERO, Armando John. 1996. Suelos. Scientia Guianae, 6: 44-47.

GONZALEZ, Juan. 2014. La corrupción campea en el Caura el Paragua. Diario el Luchador. 10 de Julio de 2014.

HEINEN, Heinz Dieter; VELASQUEZ, Juan; TOMEDES, Ramon. 1981. Informe sobre la empresa indígena intercomunitaria Tujuumoto (Alto Caura, Dtt. Cedeño, Edo. Bolivar). Caracas: Instituto Agrario Nacional.

HUBER, Otto y ROSALES, Judith (eds.). 1996. Scientia Guianae No. 6. Ecología de la Cuenca del Río Caura. I Estudios Generales. Caracas: Bioguayana.

. (eds.). 1997. Scientia Guianae No. 7. Ecología de la Cuenca del Río Caura. II Estudios Especiales. Caracas: Bioguayana.

KOCH GRÜMBER, Theodor. 1979 (1917). Del Roraima al Orinoco. Ediciones del Banco Central de Venezuela. Colección Histórico-económica. Caracas. 3 vols.

HUMBOLDT, Alejandro de. 1991 (1804). Viaje a las regiones equinocciales del nuevo continente. Caracas: Monte Avila Editores. 5 vols.

MARIN, Euler y CHAVIEL, Alfredo. 1996. Bosques de tierra firme. Scientia Guianae, 6: 60-65.

MICHELENA y ROJAS, Francisco. 1987 (1867) Exploración oficial desde el norte de la América del Sur. Caracas: Editorial Gestión.

MINISTERIO de EDUCACION, CULTURA y DEPORTES (MECD). 2002. Guía Pedagógica Dhe'cwana/Ye'kwana para la Educación Intercultural Bilingüe. Dirección de Asuntos Indígenas, Zona Educativa del Estado Bolívar, Zona Educativa del Estado Amazonas. Caracas: MECD-UNICEF.

POPULAÇÃO INDÍGENA no BRASIL. Disponible en: <https://pib.socioambiental.org/es?search=yekuana\%20brasil $>$.

RED ARA. 2013. La contaminación por mercurio en la Guayana venezolana: Una propuesta de diálogo para la acción. Caracas. Disponible en: <http://red-ara-venezuela.blogspot.com>.

REPUBLICA de VENEZUELA (RDV). 1961. Constitución de la República de Venezuela. Publicada 
en Gaceta Oficial No. 662 (Extraordinario) de fecha 23 de Enero de 1961.

REPUBLICA de VENEZUELA (RDV). 1979. Decreto No. 283 para la Educación Intercultural Bilingüe. Caracas: Ministerio de Educación.

REPUBLICA BOLIVARIANA de VENEZUELA (RBV). 1999. Constitución de la República Bolivariana de Venezuela. Caracas: Asamblea Nacional.

REPUBLICA BOLIVARIANA de VENEZUELA (RBV). 2009 Ley Orgánica de los Consejos Comunales. Gaceta Oficial de la RBV No. 39.335 del 28 de diciembre de 2009. Disponible en: <www. minci.gob.ve>.

REPUBLICA BOLIVARIANA de VENEZUELA (RBV). 2011. Resultados Población Indígena. XIV Censo de Población y vivienda 2011. Gerencia General de Estadísticas Demográficas. Caracas: Instituto Nacional de Estadística INE.

REPUBLICA BOLIVARIANA de VENEZUELA (RBV). 2016. Decreto 2.248 de creación de la zona de desarrollo estratégico nacional "Arco Minero del Orinoco. Gaceta Oficial de la RBV. Caracas. Año CXLIII Mes. V Número 40855.

REPUBLICA BOLIVARIANA de VENEZUELA (RBV). 2016a . Decreto No. 2265 de creación de la Comisión Presidencial de Desarrollo Ecosocialista y Salvaguarda de los Derechos de los Pueblos Indígenas en la Actividad Minera. Caracas.

REPUBLICA BOLIVARIANA de VENEZUELA (RBV). 2017 Creación Parque Nacional Caura. Gaceta Oficial de la RBV. Caracas. Año CXLIV-Mes VI. Número 41.118.

SILVA MONTERREY, Nalua Rosa. 1992. La constitución du groupe local: le cas de Jyuwutunña. Mémoire du Diplome d'Etudes Approfondies (DEA) en Anthropologie Sociale et Ethnologie. Paris: Ecole des Hautes Etudes en Scienes Sociales.

Fondo Editorial UNEG.

2006. Introducción a la Etnografía de los Pueblos Indígenas de Guayana. Puerto Ordaz:

. 2007. Pouvoir Parenté et Société. De la Diversité a la synthese. Thése de Doctorat en Athropologie Sociale et Ethnologie. Paris: Ecole des Hautes Etudes en Sciences Sociales.

. 2010. Pouvoir Parenté et Société entre lesYekwana du Caura,Vénézuela. De la Diversité a la synthese. Sarrebruck, Allemagne : Editions Universitaires Europeennes. 420 pp.

2012. (Redacción y edición) Pautas para el manejo de los hábitats ye’kwana y sanema en la cuenca del Caura. Forest Peoples Programme. 126 pp.

. 2013. Diversidad constitutiva ye'kwana. En Antropológica No. 119-120. FLASA.

p. 119-141. (disponible en línea)

2015. Poder, Parentesco y Sociedad. Entre los Ye'kwana del Caura-Venezuela. Quito: Abya-Yala. 372 pp.

. 2016. Tanüko la fiesta de las palmas entre los Ye'kwana. En Boletin Antropológico Año 34. Número 91. Enero-Junio 2016. ULA. pag. 35-52. (disponible en línea)

SILVA MONTERREY, Nalua Rosa y NUÑEZ, Coromoto. 2006. Los acontecimientos de la cuenca del río Caura”. En la Iglesia en Amazonas. Año XXVI No. 114. Diciembre. p. 20-22.

VARGAS, Horacio y RANGEL, Jose. 1996. Hidrología y sedimentos. Scientia Guianae, 6: 48-53.

WATSON, Fiona. 2016. Envenenamiento por mercurio de indígenas amazónicos: nuevos datos 
salen a la luz. Survival International. Disponible en: <http://www.survival.es/noticias/11183 $23 / 03 / 2016>$.

Resumo: A partir da minha experiência como pesquisadora entre os Ye'kwana da Bacia do Caura (fronteira com o Brasil) por mais de vinte e cinco anos, e das fontes documentais, analisarei brevemente as formas de organização do Ye'kwana e as suas relações intraétnicas e interétnicas, a fim de pôr em perspectiva a relação binacional com os governos venezuelano e brasileiro, para determinar o impacto que a fronteira tem sobre sua sociedade como um todo.

Palavras-chave: Ye'kwana, Venezuela, relações intra e interétnicas
Abstract: Based on my research for over twenty-five years among the Ye'kwana from Caura Basin (border with Brazil), and from documentary sources, I shall analyze their forms of organization and their intra- and inter-ethnic relationships, in order to observe the binational context involving the Venezuelan and Brazilian governments to examine the impact that living on the international border has on their society as a whole.

Keywords: Ye'kwana, Venezuela, intra- and inter-ethnic relationships 